INPLASY

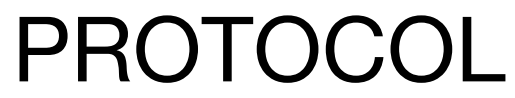

To cite: Sun et al. Comparative efficacy and safety of traditional Chinese patent medicine for NAFLD in Childhood or Adolescence A Bayesian network metaanalysis protocol. Inplasy protocol 2020120068. doi: 10.37766/inplasy2020.12.0068

Received: 12 December 2020

Published: 12 December 2020

Corresponding author:

Yuli Sun

sunyulijn@126.com

Author Affiliation:

Shandong University of Traditional Chinese Medicine

Support: Medicine project (2019-0114).

Review Stage at time of this submission: Preliminary searches.

Conflicts of interest:

None.

\section{Comparative efficacy and safety of traditional Chinese patent medicine for NAFLD in Childhood or Adolescence A Bayesian network meta-analysis protocol}

Sun, YL1; Tan, ZF2; Jiang, ZY3; Li, M4; Huang, YY5; Sun, JG6.

Review question / Objective: Chinese patent medicines play an important role in the treatment of NAFLD. At present, there is no comparison of the safety and effectiveness of various Chinese patent medicines curing NAFLD in Childhood or Adolescence. So we take advantage of the method of network meta-analysis to systematically compare the efficacy of various Chinese patent medicines curing this disease.

Information sources: We will comprehensively search the Cochrane Library, PubMed, Embase, ClinicalTrials foreign databases, and CNKI, Wanfang database, Weipu database, China Biomedical database. The search strategy will be constructed in the form of Medical Subject Headings (MeSH) combine with keywords. We will also search ongoing trial registers in the trial registry websites.

INPLASY registration number: This protocol was registered with the International Platform of Registered Systematic Review and Meta-Analysis Protocols (INPLASY) on 12 December 2020 and was last updated on 12 December 2020 (registration number INPLASY2020120068).

\section{INTRODUCTION}

Review question / Objective: Chinese patent medicines play an important role in the treatment of NAFLD. At present, there is no comparison of the safety and effectiveness of various Chinese patent medicines curing NAFLD in Childhood or
Adolescence. So we take advantage of the method of network meta-analysis to systematically compare the efficacy of various Chinese patent medicines curing this disease.

Condition being studied: Nonalcoholic fatty liver disease(NAFLD) in childhood or 
adolescence refers to the chronic fatty degeneration of the liver under 18, involving more than $5 \%$ of liver cells, drinking, and other apparent pathogenic factors that lead to chronic fatty deposits in the liver are excluded. According to the clinical and pathological characteristics, NAFLD can be divided into nonalcoholic fatty liver (NAFL), nonalcoholic steatohepatitis (NASH) and associated hepatic fibrosis and cirrhosis. According to statistics, the prevalence of NAFLD in children in the United States is about $3 \%$ to $11 \%$, which is lower than in Asia. The prevalence of NAFLD in obese and overweight children has increased significantly, reaching $50 \%$ to $80 \%$, which has become a familiar cause of chronic liver disease in children. The increasingly serious epidemic of NAFLD among children is of great concern because NAFLD is considered a more widespread and potentially metabolic manifestation of liver dysfunction and is closely associated with many metabolic risk factors, including insulin resistance, dyslipidemia, cardiovascular disease and obesity. Although it is rare to find children die of NAFLD, the risk of hepatic cirrhosis and hepatocellular carcinoma increases significantly in adulthood. Besides, it may also increase the incidence and mortality of extrahepatic diseases such as cardiocerebrovascular diseases.

\section{METHODS}

Participant or population: The population includes NAFLD in childhood or adolescence diagnosed according to any internationally or nationally authorized diagnostic criteria (such as guidelines for non-alcoholic fatty liver disease in children and adolescents; expert consensus on the diagnosis and treatment of non-alcoholic fatty liver disease in children.

Intervention: The experimental group received Chinese patent treatment alone, or Chinese patent medicine combined with conventional Western medicine treatment. Chinese patent medicines included Xiaoyao Pills, Dangfei Liganning Capsules, Huazhi
Rougan Granules, Qiao Zhi Capsules, Xuezhikang Capsules, Gynostemma Glycoside Tablets, Yinzhihuang granules, Hugan Ning tablets, Anluo Huaxian Pills, Liuwei Wuling tablets etc.; The control group received conventional Western medicine treatment, including oral medication or diet exercise treatment. RCTs that use two or more Chinese patent medicines or combined acupuncture, moxibustion and other traditional Chinese medicine methods are excluded.

Comparator: The control group received conventional Western medicine treatment, including oral medication or diet exercise treatment.

Study designs to be included: We will include all RCTs using TCPM for the treatment of NAFLD in childhood or adolescence.

Eligibility criteria: We include studies if they meet the following criteria: 1)Adolescents or children diagnosed with NAFLD according to any internationally or nationally authorized diagnostic criteria;

2) The experimental group was treated with traditional Chinese patent medicines cobined with conventional Western medicine; 3) The control group received Western medicine treatment, including oral medication or diet exercise treatment; 4)study types are randomized controlled trials.

Information sources: We will comprehensively search the Cochrane Library, PubMed, Embase, ClinicalTrials foreign databases, and CNKI, Wanfang database, Weipu database, China Biomedical database. The search strategy will be constructed in the form of Medical Subject Headings (MeSH) combine with keywords. We will also search ongoing trial registers in the trial registry websites.

Main outcome(s): The main indicators is the rate of remission of clinical symptoms after treatment; ALT continues to decrease, defined as $50 \%$ or less of the baseline level or $40 \mathrm{U} / \mathrm{L}$ or less. 
Additional outcome(s): The secondary indicators are to improve the histological characteristics of NAFLD and the resolution of NASH; the incidence of liver fibrosis, cirrhosis, and liver cancer; the incidence of diabetes and hypertension.and adverse events.

Data management: The included literature must cover one or more main indicators.

Quality assessment / Risk of bias analysis: The quality of each trial will be assessed by two researchers independently based on the Cochrane Risk of Bias Risk Assess ment Tool recmmenedby Cochrane Handbook version 5.1.0. Use the decision words "high risk", "low risk", and "unclear risk" to evaluate the quality of the input article in 7 aspects, including: whether the random sequence is sufficient; whether there is hidden allocation; whether blinding is used; whether the result data is complete; Whether there is selective reporting; whether there is publication bias; others.

Strategy of data synthesis: We will use Stata 14.0 software and Markov chainMonte Carlo (MCMC) method to conduct Bayesian meta-analysis. Three Markov chains will be used for simulation, and the number of iterations will be set at 50,000 (the first 20,000 are used for annealing to eliminate the effect of the initial value, and the last 30,000 are used for sampling). The reticular diagram will be drawn by Stata 15.0 software to show the direct and indirect comparison between different interventions. The relative odds ratio (RoR) and its $95 \%$ confidence interval (CI) are calculated to evaluate the consistency of each closed loop. The lower limit of $95 \% \mathrm{Cl}$ is equal to 1 , indicating good consistency. If $R o R$ is close to 1 , direct evidence and indirect evidence are consistent, and the fixed effect model is adopted for analysis. Otherwise, the closed-loop is considered to have obvious inconsistencies, and the random effect model is used for analysis. Dichotomous data will be represented by odds ratio (OR) and $95 \% \mathrm{Cl}$, and $\mathrm{P}<0.05$ was considered statistically significant. WinBUGS 1.4 .3 will be used to rank the efficacy of different interventions and the area under the curve will be recorded (the area under the curve will be expressed as a percentage, the larger the value, the better the effect).

Subgroup analysis: Subgroup analysis will be considered if sufficient data is available.

Sensibility analysis: Sensitivity analysis will be conducted with symptom improvement rate to evaluate clinical similarity and methodology of included studies to determine the reliability of the results of this study.

\section{Country(ies) involved: China.}

Keywords: Traditional Chinese patent medicine (TCPM), NAFLD in childhood or adolescence, Network meta-analysis, Protocol.

Contributions of each author:

Author 1 - Yuli Sun - The author drafted the manuscript.

Email: sunyulijn@126.com

Author 2 - Zhaofeng Tan - The author provided statistical expertise.

Email: tanzhaofeng@126.com

Author 3 - Zhenyuan Jiang - The author contributed to the development.

Email: 1065658987@qq.com

Author 4 - Min Li - The author read, provided feedback and approved the final manuscript.

Email: sdlm32167@163.com

Author 5 - Yaoyao Huang - The author read, provided feedback and approved the final manuscript.

Email: hyao626@163.com

Author 6 - Jianguang Sun - The author read, provided feedback and approved the final manuscript.

Email: 57369400@qq.com 